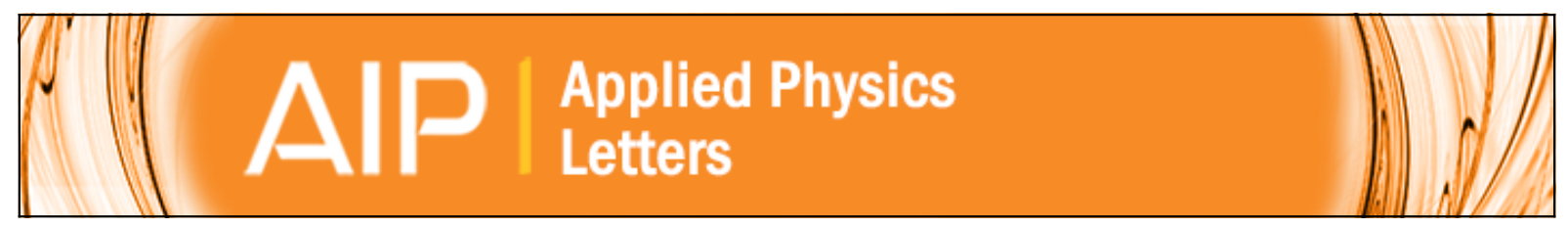

\title{
Magnetocaloric effect in random magnetic anisotropy materials
}

Xavier Bohigas, Javier Tejada, Francesc Torres, José Ignacio Arnaudas, Enrique Joven, and Agustn del Moral

Citation: Applied Physics Letters 81, 2427 (2002); doi: 10.1063/1.1506777

View online: http://dx.doi.org/10.1063/1.1506777

View Table of Contents: http://scitation.aip.org/content/aip/journal/apl/81/13?ver=pdfcov

Published by the AIP Publishing

\section{AlP}

\section{Create a profile.




\title{
Magnetocaloric effect in random magnetic anisotropy materials
}

\author{
Xavier Bohigas ${ }^{a)}$ \\ Departament Física i Enginyeria Nuclear, Universitat Politècnica de Catalunya, Diagonal 647, \\ 08028 Barcelona, Spain \\ Javier Tejada and Francesc Torres \\ Departament Física Fonamental, Universitat de Barcelona, Diagonal 647, 08028 Barcelona, Spain \\ José Ignacio Arnaudas, Enrique Joven, ${ }^{\text {b) }}$ and Agustín del Moral \\ Departament Física de la Materia Condensada, Universidad de Zaragoza, 50015 Zaragoza, Spain
}

(Received 13 February 2002; accepted for publication 19 July 2002)

In this letter we report the results of entropy variations in random anisotropy magnets composed of $\mathrm{Tb}_{x} \mathrm{Y}_{1-x} \mathrm{Al}_{2}$, with $x=0.15,0.20,0.25,0.35,0.40$, and 0.50 . We discovered large entropy variation associated with the spin glass to paramagnetic transition. Both temperature transition and entropy changes were studied at different temperatures and with different compositions. Our conclusion is that these materials are suitable candidates for use as magnetic refrigerants in a temperature range below 40 K. ㅇ 2002 American Institute of Physics. [DOI: 10.1063/1.1506777]

It is known that magnetic refrigeration is a serious and real alternative to current conventional refrigeration systems. ${ }^{1,2}$ One of the most critical factors for using magnetic refrigeration in refrigerators is that of finding a suitable material within the working temperature range of refrigeration. This accounts for the increasing interest, in recent years, in the study and characterization of magnetic materials that show large entropy changes when subjected to magnetic intensity variation.

Magnetic refrigeration is based on the magnetocaloric effect. When a magnetic material is subjected to the action of an applied magnetic field variation, an entropy change is brought about in the magnetic sublattice of the material. Since the degree of the alignment of magnetic moments present in the magnetic sublattice of the system changes when a magnetic field variation is applied, the magnetic entropy will also change. An increase in magnetic entropy will be observed when the applied magnetic field intensity is reduced, and conversely the entropy will decrease when the magnetic field increases, since the order of the magnetic moments in the material increases. The reduction in the magnetic entropy of a material when magnetic fields are applied adiabatically, $\Delta S$, causes the lattice entropy to increase by the same amount, $\Delta S$, and the temperature of the material thus increases. This is known as the magnetocaloric effect.

The magnetocaloric effect was studied for different materials in a wide temperature range. Under $40 \mathrm{~K}$ there are two types of materials that show a significant magnetocaloric effect. One of them shows paramagnetic behavior within this temperature range. ${ }^{3}$ The second class of materials are those in which one can to take advantage of the entropy change associated with the ferromagnetic to paramagnetic transition when the magnetic materials are subjected to an applied field variation near their Curie temperature. ${ }^{2,45}$ The giant and

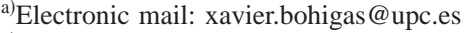

b) Permanent address: Instituto de Astrofísica de Canarias, C/ Vía Láctea, 38200 La Laguna, Tenerife, Spain.
}

time-dependent magnetocaloric effect in molecular clusters was recently studied by our group. ${ }^{6}$

In this article we present the results of entropy variation and temperature phase transition in random magnetic anisotropy materials under a modest applied magnetic field.

Measurements of magnetic entropy variation when a fixed magnetic field change is applied allow one to determine whether a magnetic material may be considered a good magnetic refrigerant. Alternatively, refrigerant capacity may be determined directly by measuring the temperature change under an applied magnetic field variation. The equivalence of these two different methods has been demonstrated. ${ }^{4,7}$

In an isothermal process of magnetization, the total magnetic entropy change of the system due to the application of a magnetic field, $\Delta S_{B}$, can be derived from Maxwell relations by integrating over the magnetic field, i.e.,

$$
\Delta S_{B}(T, B)=\int_{B_{\min }}^{B_{\max }}\left(\frac{\partial M}{\partial T}\right)_{B} d B,
$$

where $B_{\min }$ and $B_{\max }$ represent the initial and final values of the magnetic induction. ${ }^{5}$

It seems clear that a high magnetocaloric effect will be observed when the magnetization varies sharply in a constant field. This occurs, for example, at the Curie temperature for ferromagnetic-paramagnetic transitions, where the maximum absolute value of the entropy change is expected.

Samples were prepared using the argon arc melting technique. The buttons obtained were remelted several times and annealed at $800^{\circ} \mathrm{C}$ for approximately one week, in order to ensure good homogeneity and the elimination of spurious phases. This was checked using powder x-ray analysis. The magnetization measurements were taken using an induction method magnetometer. The magnetization isotherms were measured by cooling the sample down to $3 \mathrm{~K}$ in a nominally zero applied field and then applying an increasing field at the temperature of measurement. ${ }^{8}$ The magnetic properties of these materials have been studied extensively. ${ }^{9}$ 


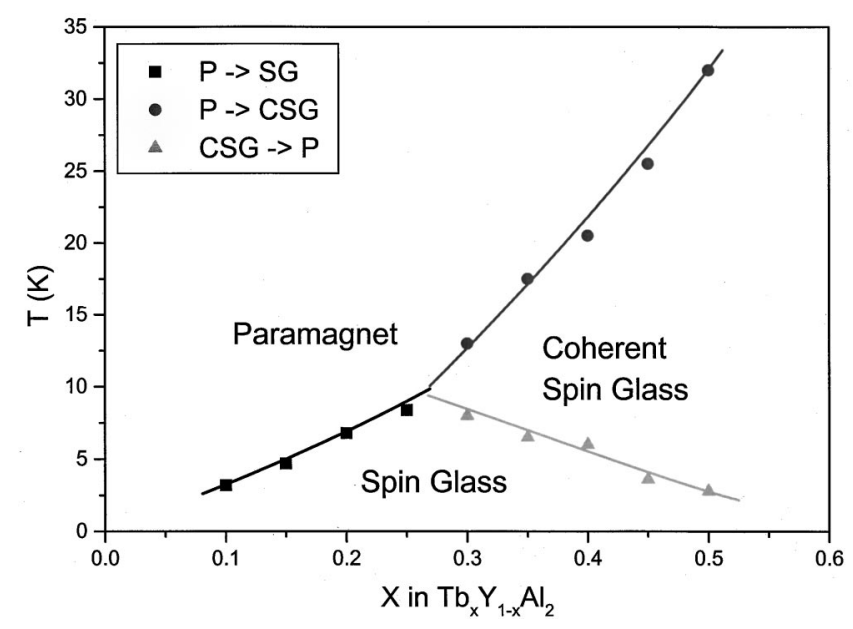

FIG. 1. Magnetic phase diagram for $\mathrm{Tb}_{x} \mathrm{Y}_{1-x} \mathrm{Al}_{2}$ compounds.

Initial ac susceptibility, $\chi_{\text {ac }}$, was measured as a function of the temperature for different compounds of the formula $\mathrm{Tb}_{x} \mathrm{Y}_{1-x} \mathrm{Al}_{2}$, with $x=0.15,0.20,0.25,0.35,0.40$, and 0.50 . The real part of $\chi_{\text {ac }}$ shows two different types of behavior depending on the Tb composition. For $x<0.30$, a sharp peak is observed at a certain temperature, $T_{\mathrm{SG}}$, which increases with composition. According to the Edwards-Anderson model, this temperature is associated with the spin glass to paramagnetic phase transition. ${ }^{10}$ For $x>0.30$, this peak transforms into a smooth shoulder, with a second maximum appearing at a higher temperature, which also increases with $x$. From the susceptibility measurements, we have proposed the phase diagram presented in Fig. 1 for these materials. ${ }^{8}$

In order to evaluate the magnetocaloric effect of our materials, we calculated the entropy changes associated with an applied magnetic field variation from the isotherm curves of magnetization versus the applied field by using the expression

$$
\left|\Delta S_{B}\right|=\sum_{i} \frac{M_{i}-M_{i+1}}{T_{i}-T_{i+1}} \cdot \Delta B_{i},
$$

where $M_{i}$ and $M_{i+1}$ are the experimental values of magnetization at $T_{i}$ and $T_{i+1}$ temperatures, respectively, under an applied field of intensity $B_{i}$. This expression is a numerical approximation to the integral in Eq. (1). Figure 2 shows isothermal magnetization measurements at different temperatures corresponding to the sample $\mathrm{Tb}_{0.50} \mathrm{Y}_{0.50} \mathrm{Al}_{2}$.

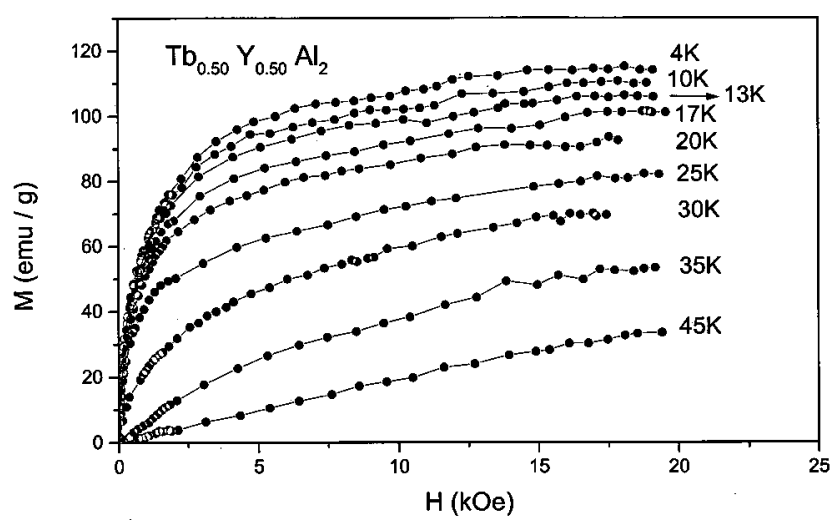

FIG. 2. Entropy change was calculated from experimental magnetization curves vs applied magnetic field.

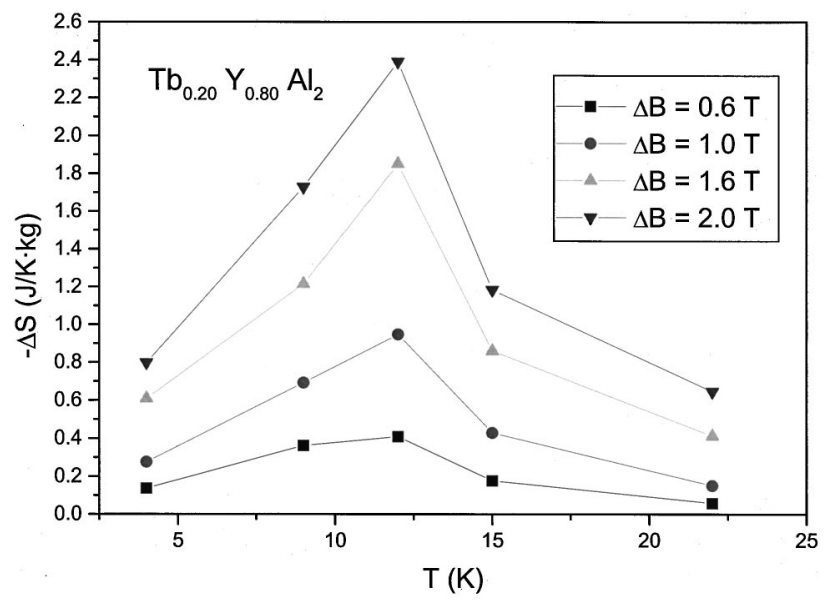

FIG. 3. Temperature dependence of entropy changes corresponding to the compound $\mathrm{Tb}_{0.20} \mathrm{Y}_{0.80} \mathrm{Al}_{2}$.

Figures 3 and 4 show the temperature dependence of entropy change corresponding to the compounds $\mathrm{Tb}_{0.20} \mathrm{Y}_{0.80} \mathrm{Al}_{2}$ and $\mathrm{Tb}_{0.50} \mathrm{Y}_{0.50} \mathrm{Al}_{2}$, respectively, similar behavior is observed for the other samples studied. There is a clear increase in the entropy change of the specimen when the temperature increases up to temperature $T_{\max }$, at which a peak appears, with falling entropy change for temperatures above $T_{\max }$. This temperature peak agrees well for all compositions, with the temperature at which the spin glass to paramagnetic transition occurs. The large entropy change associated with this phase transition can be explained by the magnetic order of these two different magnetic phases. Magnetic moments are partially ordered in the coherent spin glass phase, whereas in the paramagnetic phase the magnetic moments are disordered because there is no interaction between them. Thus, in accordance with this change in the magnetic order, an entropy change is expected at the magnetic phase transition temperature.

The entropy variation changes considerably according to the composition, as can be seen in Table I, which shows the entropy variation calculated for different applied magnetic fields. When the $\mathrm{Tb}$ composition increases, the entropy change likewise increases, reaching a value of $7.6 \mathrm{~J} / \mathrm{K} \mathrm{kg}$ corresponding to the $\mathrm{Tb}_{0.5} \mathrm{Y}_{0.5} \mathrm{Al}_{2}$ sample when a modest magnetic field variation of $2 \mathrm{~T}$ is applied. Samples exhibit different transition temperatures when the material composi-

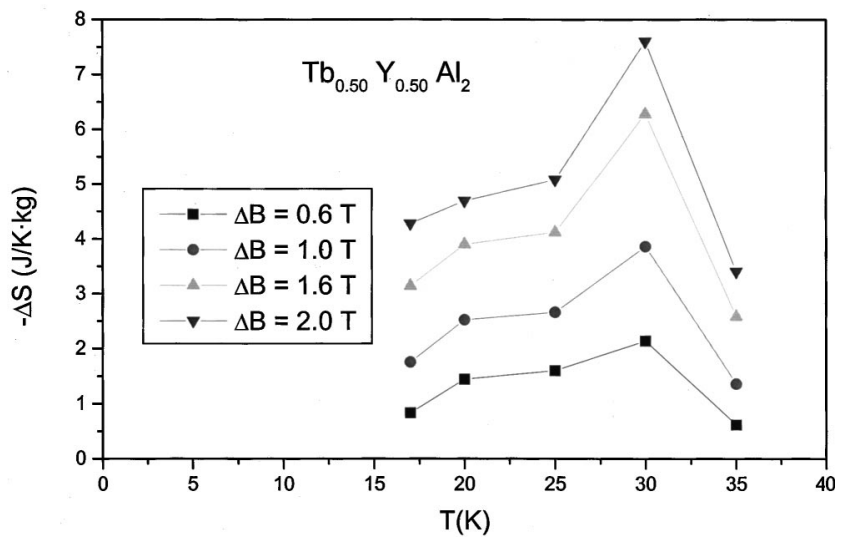

FIG. 4. Temperature dependence of entropy changes corresponding to the compound $\mathrm{Tb}_{0.50} \mathrm{Y}_{0.50} \mathrm{Al}_{2}$. 
TABLE I. Absolute values of the entropy variation calculated from the isotherm magnetization curves of our materials when different magnetic field intensity variation is applied. The table also shows the temperature at which the curve $|\Delta S(\mathrm{~T})|$ shows a maximum for different compositions of $\mathrm{Tb}_{x} \mathrm{Y}_{1-x} \mathrm{Al}_{2}$.

\begin{tabular}{cccccc}
\hline \hline $\begin{array}{c}X \\
\text { in } \mathrm{Tb}_{x} \mathrm{Y}_{1-x} \mathrm{Al}_{2}\end{array}$ & $\begin{array}{c}|\Delta S|(\mathrm{J} / \mathrm{kg} \mathrm{K}) \\
(\Delta B=0.6 \mathrm{~T})\end{array}$ & $\begin{array}{c}|\Delta S|(\mathrm{J} / \mathrm{kg} \mathrm{K}) \\
(\Delta B=1.0 \mathrm{~T})\end{array}$ & $\begin{array}{c}|\Delta S|(\mathrm{J} / \mathrm{kg} \mathrm{K}) \\
(\Delta B=1.6 \mathrm{~T})\end{array}$ & $\begin{array}{c}|\Delta S|(\mathrm{J} / \mathrm{kg} \mathrm{K}) \\
(\Delta B=2.0 \mathrm{~T})\end{array}$ & $\begin{array}{c}T_{\max } \\
(\mathrm{K})\end{array}$ \\
\hline 0.15 & 0.3 & 0.4 & 0.9 & 1.2 & 10 \\
0.20 & 0.4 & 1.0 & 1.8 & 2.4 & 12 \\
0.25 & 0.5 & 1.1 & 1.9 & 2.5 & 14 \\
0.35 & 1.0 & 1.8 & 2.8 & 3.5 & 16 \\
0.40 & 0.9 & 2.9 & 3.8 & 4.5 & 22 \\
0.50 & 2.1 & 3.9 & 6.3 & 7.6 & 30 \\
\hline \hline
\end{tabular}

tion varies within the $10-30 \mathrm{~K}$ temperature range, as can be seen in Table I. A similar dependence of entropy change on composition has been observed in other families of materials, such as perovskite structures with the formula $\mathrm{La}_{0.65} \mathrm{Ca}_{0.35} \mathrm{Ti}_{1-x} \mathrm{Mn}_{x} \mathrm{O}_{3}$ (Ref. 11) and series of pseudobinary $\mathrm{Dy}_{1-x} \mathrm{Er}_{x} \mathrm{Al}_{2}$ compounds. ${ }^{2,12}$

Calculated entropy variations under modest applied fields for different compositions of $\mathrm{Yb}$ in our materials are summarized in Table I. The entropy change values are greater than, or at least similar to, other previously published results calculated in ferromagnetic to paramagnetic phase transitions in the same temperature ranges. The maximum value of the entropy variation detected in our materials is 7.6 $\mathrm{J} / \mathrm{Kg} \mathrm{K}$ for a magnetic field change of $2 \mathrm{~T}$. This value is higher than that of previously published results ${ }^{12}$ for similar aluminides with the same field variation, $\sim 5 \mathrm{~J} / \mathrm{kg} \mathrm{K}$ (for $\mathrm{ErAl}_{2}$ ), $\sim 2.1 \mathrm{~J} / \mathrm{kgK}$ (for $\mathrm{DyAl}_{2}$ ), and $\sim 3 \mathrm{~J} / \mathrm{kg} \mathrm{K}$ (for $\mathrm{DyNi}_{2}$ ), and also for the families of materials with the formula $\mathrm{Dy}_{1-x} \mathrm{Er}_{x} \mathrm{Al}_{2}$, which show entropy changes of 2-5 $\mathrm{J} / \mathrm{kg} \mathrm{K}$ (Ref. 2) in a similar temperature range (10-60 K). As shown recently by Pecharsky and Gschneidner, ${ }^{4}$ a meaningful comparison of entropy change in different materials should be made using $\mathrm{J} / \mathrm{mol}$ (atom) $\mathrm{K}$, and not $\mathrm{J} / \mathrm{kg} \mathrm{K}$, i.e., by presenting the entropy change for 1 mole of atoms, and not for unit mass (or volume). Accepting this point of view, the $\mathrm{Tb}_{0.5} Y_{0.5} \mathrm{Al}_{2}$ aluminate shows an entropy change of 0.45 $\mathrm{J} / \mathrm{mol}$ (atom) $\mathrm{K}$ for $\Delta B=2 \mathrm{~T}$, while the entropy variation for the $\mathrm{Dy}_{1-x} \mathrm{Er}_{x} \mathrm{Al}_{2}$ compounds increases from 0.15 to 0.40 $\mathrm{J} / \mathrm{mol}$ (atom) $\mathrm{K}$ for the same field variation and a similar temperature range.

Table I also shows the temperature at which the entropy variation is at a maximum. These values correspond quite well with those of the spin glass to paramagnetic transition presented in Fig. 1. This indicates that the phase transition temperature corresponds to the temperature at which maximum entropy variation is achieved. It is clear from Table I that $T_{\max }$ increases over the $10-30 \mathrm{~K}$ temperature range and that some of these materials show a sufficient magnetocaloric effect to be used as active magnetic refrigerants within this temperature range.

In conclusion, we have shown data for the magnetic component of entropy variation in random anisotropy magnets as a function of the applied field, temperature, and composition. These data clearly indicate that the magnetocaloric effect based on spin glass to paramagnetic phase transition in $\mathrm{Tb}_{x} \mathrm{Y}_{1-x} \mathrm{Al}_{2}$ materials is high and tunable. The results obtained show a strong dependence on the composition of the material. These materials are therefore suitable candidates for use as magnetic refrigerants within a temperature range below $40 \mathrm{~K}$.

X.B., J.T., and F.T. would like to thank MATGAS for financial support.

${ }^{1}$ G. V. Brown, J. Appl. Phys. 47, 3673 (1976).

${ }^{2}$ K. A. Gschneidner, Jr. and V. K. Pecharsky, Annu. Rev. Mater. Sci. 30, 387 (2000), and references therein.

${ }^{3}$ J. A. Barclay, D. Moze, and L. Patterson, J. Appl. Phys. 50, 50870 (1979); R. D. Shull, R. D. McMichael, and J. J. Ritter, Nanostruct. Mater. 2, 205 (1993); H. Kimura, T. Numazawa, M. Sato, T. Ikoya, and T. Fukuda, J. Appl. Phys. 77, 432 (1995).

${ }^{4}$ V. K. Pecharsky and K. A. Gschneidner, Jr., J. Appl. Phys. 90, 4614 (2001), and references therein.

${ }^{5}$ X. Bohigas, J. Tejada, E. del Barco, X. X. Zhang, and M. Sales, Appl. Phys. Lett. 73, 390 (1998).

${ }^{6}$ F. Torres, J. M. Hernández, X. Bohigas, and J. Tejada, Appl. Phys. Lett. 77, 3248 (2000).

${ }^{7}$ X. Bohigas, J. Tejada, M. L. Martínez-Sarrión, S. Tripp, and R. Black, J. Magn. Magn. Mater. 208, 85 (2000).

${ }^{8}$ E. Joven, A. del Moral, and J. I. Arnaudas, J. Appl. Phys. 69, 5069 (1991).

${ }^{9}$ A. del Moral, J. I. Arnaudas, C. de la Fuente, M. Ciria, and E. Joven, J. Appl. Phys. 76, 6180 (1994), and references therein.

${ }^{10}$ S. F. Edwards and P. J. Anderson, J. Phys. E 11, 965 (1978).

${ }^{11}$ X. Bohigas, E. del Barco, M. Sales, and J. Tejada, J. Magn. Magn. Mater. 196-197, 455 (1999).

${ }^{12}$ P. J. von Ranke, V. K. Pecharsky, and K. A. Gschneidner, Jr., Phys. Rev. B 58, 12110 (1998). 